\title{
Pricing Spectrum Access in Cellular CDMA Networks with Heterogeneous Demand (Invited Paper)
}

\author{
Ashraf Al Daoud, Murat Alanyali, and David Starobinski \\ Department of Electrical and Computer Engineering \\ Boston University, Boston, MA 02215 \\ Email: $\{$ ashraf, alanyali, staro\}@bu.edu
}

\begin{abstract}
We consider pricing secondary access to wireless spectrum in cellular CDMA networks. We study the case for a primary license holder interested in leasing the right of providing service in a given geographical region of its coverage network. The goal is to price access to the cells in that region under heterogeneous call traffic demand with the objective of profit maximization. While a revenue is gained from the leased region due to the exercised price, the primary license holder incurs a loss due to reduced spatial coverage of the network and also due to interference effect from the leased into the retained region. We exploit the spatial effect of interference due to geographical locations of the cells and set a price per cell rather than pricing the whole region by a scalar quantity. We employ reduced load approximations which have proved useful in classical telephony and characterize optimal prices for different pricing philosophies, e.g., flat pricing and demand-based pricing. The obtained formula of prices suggests charging per admitted call in proportion with the interference that the call generates. The charged amount balances the corresponding loss of revenue due to the influence of an admitted call. We present an iterative price computing technique and provide a numerical study in support of our analytical results.
\end{abstract}

\section{INTRODUCTION}

As wireless communications become more ubiquitous, the demand for frequency spectrum is in rapid increase. Recent studies has shown that many frequency bands that have been already assigned are in fact underutilized [1]. Therefore there is a growing interest in improving the efficiency of spectrum use and introducing regulatory reforms. In this direction, the Federal Communication Commission (FCC) has lately adopted a set of policies and procedures to enable primary license holders to lease spectrum bands, paving the way for the creation of spectrum secondary markets [2]. In fact, and besides being dealing with a valuable commodity, spectrum market regulators need to guarantee property rights in light of the possibility of spatial interactions among service providers caused by the electromagnetic interference. Therefore efficient pricing lies among the major issues to be handled in this respect.

We consider the problem of spectrum pricing in cellular CDMA networks. In particular we focus on the problem of a primary license holder, or in short a licensee, which aims to abandon a subset of its coverage area and lease the right to provide service to some secondary users, call them the lessees.
We study the pricing problem from the perspective of the licensee for the purposes of maximizing its profit from the lease. In fact, and while the licensee obtains a revenue due to the exercised price, it incurs a loss due to (i) reduced spatial coverage of its network and (ii) possible interference from the leased into the retained region of its network.

While it seems natural to deal with the problem within the framework of monopolistic pricing in microeconomic theory [3], it is the network-wide effect of interference that makes it hard to come up with explicit solutions for the problem. Namely, a call in progress on a certain cell produces some interference that affects the occupancy of some neighboring cells due to geographical proximity. In the same time, a reduction in the number of calls in those cells might help some other neighboring cells to accommodate more calls, and the effect goes further and further. This knock-on effect is also applicable to every cell in the network. One approach to avoid dealing with such complexities is to isolate the secondary traffic in the network by using guardbands [4]. A guardband, however, is an unutilized resource whose cost needs to be internalized either by the licensee or by the lessee. The situation leads to an inevitable loss of efficiency in the transaction which may in fact be significant. The attendant inefficiency in turn limits the granularity and thereby liquidity of a secondary spectrum market.

In this work, we consider optimal pricing of spectrum in light of heterogenous call traffic demand and without resorting to conservative methods to eliminate interference. We employ reduced load approximations which have found application in classical telephony to estimate blocking probabilities. The form of optimal price suggests charging the lessee per admitted call that generates interference for the licensee. The charged amount is shown to depend on the extent of generated interference, namely, it balances the corresponding loss of revenue incurred by the licensee due to the influence of an admitted call. This effort entails convenient analytical techniques that avoid the alluded difficulties associated with network-wide effects of interference at the expense of reasonable loss of modeling accuracy. We exploit the structure of the prices to devise an iterative procedure to compute optimal prices. Towards the end of this work we present numerical study in support of our analytical results. 


\section{RELATED WORK AND SUMMARY OF CONTRIBUTIONS}

Pricing for communication networks is a well-studied subject. See [5] for related topics and references in this area. However, the setting considered in this work is in fact specific to secondary cellular wireless markets. In related work, [6] pursues interference based pricing in a single cell via adaptive optimization techniques, and [7], [8] adopt a performance oriented viewpoint in considering dynamic spectrum access within a cell. In [9] the authors do power control by pricing transmitted power in multicell wireless data systems, while in [10] the authors use some auction mechanisms for sharing spectrum access subject to some interference constraints.

Main contributions of the present paper are

1. Global consideration of network: We consider general network topologies rather than a single cell. Rather than lumping any portion of the network into an approximate module, the paper accounts for sophisticated dependence between cells due to generated interference.

2. Characterization of optimal prices: We characterize the form of optimal prices under a general framework. Optimal prices are shown to have an interpretation that offers insight on dominant factors that in turn help determine the value of spectrum under spatial interactions.

The work presented in this paper is a direct extension to the approach pursued in our previous work [11]. In this work we set a price for each cell in the region considered for lease rather than pricing the whole region by a scalar quantity. This way we exploit the fact that the netwok-wide effect of interference due to an admitted call in one cell depends on the location of the cell in the network. Therefore we have more degrees of freedom to assign prices and consequently obtain more profit from the transaction.

The structure of the paper is as follows: The teletraffic operational model for CDMA networks is given in Section III. The formulation of the profit maximization problem is the subject of Section IV. In Section V reduced load approximations are employed and the forms of optimal prices are given in Section VI. An iterative technique for computing optimal prices is suggested in Section VII. In Section VIII a numerical study is provided in support of our analytical results. The paper concludes with final remarks in Section IX.

\section{NeTWORK OPERATIONAL MODEL}

In this section an operational model for cellular CDMA networks is introduced. In fact a network is represented with a weighted graph $G=(N, W)$ where $N$ refers to the set of nodes and $W$ refers to the set of positive edge weights. Each node $i \in N$ in the graph represents a cell. For each pair $i, j$ of cells the associated weight $w_{i j} \in W$ is a measure of inter-cell electromagnetic interference due to geographical proximity. Self-loops are in fact allowed, and it will consistently be the case that $w_{i i}>0$. The example of Figure 1 illustrates the graphical representation of a 19-cell hexagonal lattice topology.

Let $n_{i}$ be the number of calls in progress at each cell $i$ and let $\mathbf{n}$ denote the cell loads $\left(n_{i}: i \in N\right)$. A call is subject

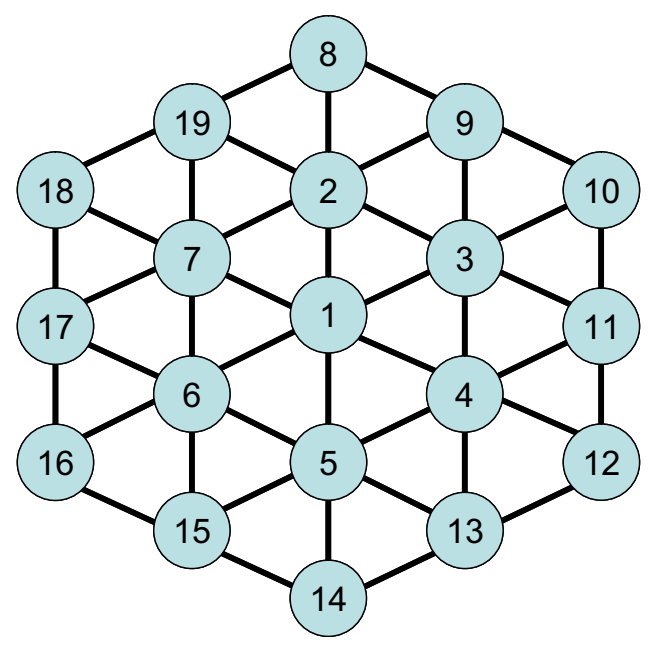

Fig. 1. The network graph of a 19-cell hexagonal lattice topology.

to interference from other calls in the same cell, as well as from calls in other cells in proportion with the associated weights. We shall assume that a call can be sustained only if it experiences small enough interference. A cell, however, may receive unbounded interference if it does not accommodate a call. A network load $\mathbf{n}$ is thus feasible if for all cells $j$ and certain constants $\kappa_{j}>0$

$$
\sum_{i \in N} n_{i} w_{i j} \leq \kappa_{j} \text { whenever } n_{j}>0 .
$$

Network models based on similar constraints have been considered in earlier works on cellular wireless CDMA networks. See, for example, [12] for an in-depth discussion of this model and specifications of model parameters $w_{i j}$ and $\kappa_{j}$ in terms of physical layer parameters and desired quality of service thresholds. In fact we shall assume that the model parameters satisfy the following mild condition:

Assumption 1: For all $i, j \in N$ the parameters $w_{i j}$ and $\kappa_{j}$ are rational numbers. Hence, without loss of generality in the feasibility condition (1), these parameters are further taken as integers.

We adopt the following statistical traffic model where calls arrive at each cell $i$ according to a Poisson process of rate $\nu_{i} \geq 0$. Arrival processes for different cells are mutually independent. Each call has a holding time that is exponentially distributed with unit mean, independently of the history prior to its arrival. An incoming call is accepted if and only if its inclusion in the network conserves the feasibility condition (1) and the call is blocked otherwise. We denote the vector of call arrival rates by $\boldsymbol{\nu}=\left(\nu_{i}: i \in N\right)$ and define $B_{i}(\boldsymbol{\nu})$ as the associated probability of call blocking on cell $i$.

The network provider generates unit revenue per admitted call in the network. We denote by $R(\boldsymbol{\nu})$ the long-term average rate of revenue generation of the provider per unit time, which is given by

$$
R(\boldsymbol{\nu})=\sum_{i \in N}\left(1-B_{i}(\boldsymbol{\nu})\right) \nu_{i} .
$$


The form of the revenue in fact admits dependence on network arrival rates $\boldsymbol{\nu}$ through cell blocking probabilities. This implicit dependence is attributed to the effect of networkwide propagation of call interference previously described as the knock-on effect. As will become clearer from the following sections, it turns out that a good handling of the pricing problem requires more insight on this dependence.

\section{Profit Maximization Problem}

We consider the problem of the primary license holder, or the licensee, for pricing cells comprising the region $L \subset N$. Namely we seek optimal cell price in leasing the right to provide service for region $L$. We denote the price for cell $i \in L$ by $p_{i}$ so that $\mathbf{p}=\left(p_{i}: i \in L\right)$. The unit of $p_{i}$ is determined by the pricing philosophy adopted by the licensee. For example if a flat price is employed then the unit of $p_{i}$ is currency per unit time, whereas for a usage-based price $p_{i}$ can be expressed in currency per Erlang.

A cell price set by the licensee invokes certain call demand from the lessee. Namely, for a given value of $p_{i}$ the call demand per unit time on cell $i$ is given by the non-increasing function $\alpha_{i}\left(p_{i}\right)$. In this work we assume the cell price to affect solely the demand on that cell. A more general version would take into consideration the effect of other cell prices on local demand. Also we assume that the call demand in the retained region $N-L$ after the transaction remains unaltered. Therefore the vector of overall network demand after a transaction at price $\mathbf{p}$, denoted by $\boldsymbol{\lambda}(\mathbf{p})=\left(\lambda_{i}\left(p_{i}\right): i \in N\right)$, has its components given as

$$
\lambda_{i}\left(p_{i}\right)= \begin{cases}\alpha_{i}\left(p_{i}\right) & \text { if } i \in L \\ \nu_{i} & \text { if } i \in N-L .\end{cases}
$$

The licensee has an expected rate of revenue over the term of the lease signed at price $\mathbf{p}$. To keep the discussion general we succinctly denote this value by $F(\mathbf{p})$. For the special cases alluded earlier in this section $F(\mathbf{p})$ may take the following forms:

a) Flat price: A flat price would be taken if it does not exceed the valuation of the commodity by potential lessees. Suppose that the licensee's apriori perception of the market value of the spectrum in cell $i$ can be represented by a random variable $V_{i}$. The licensee's expected rate of revenue generation from region $L$ by an exercised flat price $\mathbf{p}$ would then be given by

$$
F(\mathbf{p})=\sum_{i \in L} p_{i} P\left(V_{i}>p_{i}\right)
$$

b) Price per demand: The licensee may price the spectrum per unit demand generated in the region $L$, in which case $p_{i}$ refers to the revenue of the licensee per call request in cell $i$ in the region $L$ after the sale. The revenue rate $F(\mathbf{p})$ of the licensee would then be

$$
F(\mathbf{p})=\sum_{i \in L} \alpha_{i}\left(p_{i}\right) p_{i}
$$

c) Price per honored demand: Alternatively, the licensee may choose to tax the interference that the lessee generates on the former's (retained) network. This abstract principle may be interpreted as imposing a tax $p_{i}$ per accepted call in cell $i$ in the region $L$, thereby entitling the licensee to a certain share of the lessee's revenue. The rate of revenue from the lease would then be given by

$$
F(\mathbf{p})=\sum_{i \in L}\left(1-B_{i}(\boldsymbol{\lambda}(\mathbf{p}))\right) \alpha_{i}\left(p_{i}\right) p_{i} .
$$

On the other hand, a call in progress on a cell in the region $L$ affects call occupancy on other cells in the network including possibly cells in the retained region. Therefore the expected revenue from that region is affected. Namely, for each nonnegative vector $\lambda$ of arrival rates the long term revenue from the retained region can be written as

$$
Q(\boldsymbol{\lambda})=\sum_{i \in N-L}\left(1-B_{i}(\boldsymbol{\lambda})\right) \nu_{i}
$$

where the effect of the demand in region $L$ is captured by the blocking probabilities $B_{i}(\boldsymbol{\lambda})$. In particular $Q(\boldsymbol{\lambda}(\mathbf{p}))$ is the revenue of the licensee from the retained region $N-L$ after leasing region $L$ provided that the transaction is realized at the price $\mathbf{p}$.

In summary, the losses that the licensee incurs due to this transaction are due to:

1) Reduced coverage of the served network after leasing region $L$.

2) Interference from the leased to the retained region due to interference effect.

The two components can be basically captured by the quantity

$$
C(\mathbf{p})=R(\boldsymbol{\nu})-Q(\boldsymbol{\lambda}(\mathbf{p})) .
$$

Therefore we seek an optimal price vector $\mathbf{p}^{*}$ to solve the problem:

$$
\max _{\mathbf{p}}(F(\mathbf{p})-C(\mathbf{p})) .
$$

In characterizing solutions of this problem we shall assume that the following technical condition holds:

Assumption 2: The functions $F$ and $\alpha_{i}, i \in L$, are differentiable.

In fact the discussion in the next section establishes that the blocking probabilities $B_{i}(\cdot)$ are also differentiable. Hence, in light of Assumption 2, the profit $F(\mathbf{p})-C(\mathbf{p})$ is differentiable in the components of $\mathbf{p}$ and a solution $\mathbf{p}^{*}$ to the licensee's problem (4) satisfies for all $i \in L$

$$
\left.\frac{\partial}{\partial p_{i}} F(\mathbf{p})\right|_{\mathbf{p}=\mathbf{p}^{*}}=\left.\frac{\partial}{\partial p_{i}} C(\mathbf{p})\right|_{\mathbf{p}=\mathbf{p}^{*}}=-\left.\frac{\partial}{\partial p_{i}} Q(\boldsymbol{\lambda}(\mathbf{p}))\right|_{\mathbf{p}=\mathbf{p}^{*}}
$$

In this paper we seek insight on the nature of optimal prices by focusing on characterizing solutions of the first-order conditions (5). Existence and uniqueness of a solution depend on further properties of the objective function; in principle a second order analysis may be employed to obtain conditions under which (4) has a unique solution. That direction is not pursued in the present paper beyond assuming existence of a solution. 


\section{REDUCED LOAD APPROXIMATION FOR BLOCKING PRobabilities}

In this section we employ a "reduced load approximation," which has proved useful in analysis of blocking probabilities in circuit switched networks [13]. First, based on the operational model given in Section III consider the state space of feasible cell loads given by

$$
S=\left\{\mathbf{n} \in \mathbb{Z}_{+}^{|N|}: \mathbf{n} \text { satisfies condition (1) }\right\} .
$$

Note that given any arrival rates $\boldsymbol{\lambda}$ the vector of cells loads evolves according to a Markov process whose states belong to $S$. This process is obtained by truncating the state space of a reversible process that corresponds to cells loads when interference limitations are ignored; in particular its equilibrium distribution $\pi_{\boldsymbol{\lambda}}$ is given by

$$
\boldsymbol{\pi}_{\boldsymbol{\lambda}}(\mathbf{n})=G \prod_{i \in N} \frac{\lambda_{i}^{n_{i}}}{n_{i} !}, \quad \mathbf{n} \in S,
$$

where $G$ is a constant which ensures that $\boldsymbol{\pi}_{\boldsymbol{\lambda}}$ is a probability vector.

Let $\mathbf{e}(i)=\left(e_{j}(i): j \in N\right)$ be such that $e_{j}(i)=1$ if $j=i$ and $e_{j}(i)=0$ otherwise. The blocking probabilities can then be expressed as

$$
B_{i}(\boldsymbol{\lambda})=\sum_{\mathbf{n}: \mathbf{n}+\mathbf{e}(i) \notin S} \boldsymbol{\pi}_{\boldsymbol{\lambda}}(\mathbf{n}) .
$$

However despite the appealing form, further manipulation of the above expression is hindered by difficulties in computing the normalization constant $G$.

Reduced load approximation: We shall approximate $B_{i}(\boldsymbol{\lambda})$ by the quantity $\hat{B}_{i}(\boldsymbol{\lambda})$ defined by

$$
\hat{B}_{i}(\boldsymbol{\lambda})=1-\prod_{j \in N}\left(1-b_{j}(\boldsymbol{\lambda})\right)^{w_{i j}}
$$

where the numbers $b_{j}(\boldsymbol{\lambda}), j \in N$, satisfy the equalities

$b_{j}(\boldsymbol{\lambda})=E\left(\left(1-b_{j}(\boldsymbol{\lambda})\right)^{-1} \sum_{i \in N} w_{i j} \lambda_{i} \prod_{k \in N}\left(1-b_{k}(\boldsymbol{\lambda})\right)^{w_{k i}}, \kappa_{j}\right)$

and $E(\cdot, \cdot)$ denotes the Erlang blocking formula

$$
E(x, y)=\left(\sum_{m=0}^{y} \frac{x^{m}}{m !}\right)^{-1} \frac{x^{y}}{y !}
$$

for all $x>0$ and positive integer $y$. The set of equations (7) has a unique solution [13]; hence the approximation is welldefined, and furthermore the solution is differentiable in $\boldsymbol{\lambda}[14$, Lemma 2.2]. The reduced load approximation above can be better motivated by first replacing the feasibility condition (1) by

$$
\sum_{i \in N} n_{i} w_{i j} \leq \kappa_{j} .
$$

Note that this condition is more stringent than (1) in the sense that it limits the interference on idle cells as well. Under the feasibility condition (8), $\kappa_{j}$ can be regarded as capacity of cell $j$ and $w_{i j}$ can be regarded as the units of capacity reserved from cell $j$ per call in progress in cell $i$. The expression (6) then suggests that $\hat{B}_{j}(\boldsymbol{\lambda})$ is the blocking probability at cell $i$ in a hypothetical model where each unit of capacity is available independently with probability $1-b_{j}(\boldsymbol{\lambda})$ at link $j$, and furthermore availability of capacity is independent from link to link. Such a model is consistent only if the parameters $b_{j}(\boldsymbol{\lambda})$ satisfy the fixed-point relation (7).

The approximate blocking probabilities $\hat{B}_{j}(\boldsymbol{\lambda})$ are known to be asymptotically exact for the feasibility condition (8) along a limiting regime where the network arrival rates $\lambda_{j}$ and thresholds $\kappa_{j}$ increase in proportion [13]. While condition (8) leads to higher blocking than condition (1), the disparity may arguably be expected to vanish in the same limiting regime as increasing the arrival rates reduces the chances of finding cells at idle state.

\section{Vi. Characterization of Optimal Prices}

In this section we employ the reduced load approximation introduced in the previous section to obtain insightful forms of optimal prices. In fact the conclusions of this section are valid under the following simplifying assumption:

Assumption 3: (Exactness of reduced load approximation) $B_{i}(\boldsymbol{\lambda})=\hat{B}_{i}(\boldsymbol{\lambda})$ for each cell $i$ and all call arrival rates $\boldsymbol{\lambda}=$ $\left(\lambda_{i}: i \in N\right)$.

Theorem 6.1: Under Assumption 3 an inner solution $p_{i}^{*}$ of the licensee's problem (4) satisfies

$$
p_{i}^{*}=\left(1-\hat{B}_{i}\left(\boldsymbol{\lambda}\left(\mathbf{p}^{*}\right)\right)\right) \alpha_{i}\left(p_{i}^{*}\right) \gamma_{i}\left(\mathbf{p}^{*}\right)
$$

where

$$
\gamma_{i}\left(\mathbf{p}^{*}\right)=\frac{\varepsilon_{i}\left(p_{i}^{*}\right)}{\left.\frac{\partial F(\mathbf{p})}{\partial p_{i}}\right|_{\mathbf{p}=\mathbf{p}^{*}}} \sum_{j \in N} w_{i j} \frac{d}{d \kappa_{j}} Q\left(\boldsymbol{\lambda}\left(\mathbf{p}^{*}\right)\right)
$$

and $\varepsilon_{i}\left(p_{i}^{*}\right)=\frac{p_{i}^{*} \alpha_{i}^{\prime}\left(p_{i}^{*}\right)}{\alpha_{i}\left(p_{i}^{*}\right)}$ is the price elasticity of demand in cell $i$.

Theorem 6.1 can be interpreted for the three pricing philosophies alluded in Section IV as follows:

Flat price: The form (9) suggests that optimal flat price per unit time for cell $i$ is the same as the revenue generated from the cell per unit time by charging each admitted call in the cell an amount $\gamma_{i}\left(\mathbf{p}^{*}\right)$. In parsing the expression (10) for this quantity it is helpful to interpret $\frac{d}{d \kappa_{j}} Q\left(\boldsymbol{\lambda}\left(\mathbf{p}^{*}\right)\right)$ as the reduction in the licensee's revenue from the retained region $N-L$ due to unit reduction in the interference threshold of cell $j$, or equivalently due to imposing unit interference on cell $j$. An accepted call in cell $i \in L$ then leads to a reduction of $w_{i j} \frac{d}{d \kappa_{j}} Q\left(\boldsymbol{\lambda}\left(\mathbf{p}^{*}\right)\right)$ in licensee's revenue. The form (10) in turn indicates that the per-call price $\gamma_{i}\left(\mathbf{p}^{*}\right)$ balances the attendant loss of revenue, up to a multiplicative quantity that depends on the price elasticity of demand in cell $i$ and the revenue function $F$.

Price per demand: If the licensee's revenue is given by (2) then for all $i \in L$

$$
\frac{\partial F(\mathbf{p})}{\partial p_{i}}=\alpha_{i}\left(p_{i}\right)\left(1+\varepsilon_{i}\left(p_{i}\right)\right)
$$


and rearrangement of equalities (9) and (10) yields

$$
\begin{aligned}
p_{i}^{*}= & \left(1-\hat{B}_{i}\left(\boldsymbol{\lambda}\left(\mathbf{p}^{*}\right)\right)\right)\left(1+\varepsilon_{i}^{-1}\left(p_{i}^{*}\right)\right)^{-1} \times \\
& \sum_{j \in N} w_{i j} \frac{d}{d \kappa_{j}} Q\left(\boldsymbol{\lambda}\left(\mathbf{p}^{*}\right)\right) .
\end{aligned}
$$

In particular the optimal per-demand cell $i$ price $p_{i}^{*}$ is proportional to the marginal cost of the licensee due to an accepted call, discounted at rate equal to acceptance probability.

Price per honored demand: In the case when the licensee's revenue is given by (3) a relatively more explicit characterization of $\mathbf{p}^{*}$ can be obtained by defining $U(p)$ as the overall revenue of the licensee after the transaction at price $\mathbf{p}$. That is,

$$
U(\mathbf{p})=\sum_{i \in N}\left(1-\hat{B}_{i}(\boldsymbol{\lambda}(\mathbf{p}))\right) \lambda_{i}\left(p_{i}\right) r_{i}\left(p_{i}\right)
$$

where

$$
r_{i}\left(p_{i}\right)= \begin{cases}p_{i} & \text { if } i \in L \\ 1 & \text { if } i \in N-L\end{cases}
$$

and in turn

$$
F(\mathbf{p})-C(\mathbf{p})=U(\mathbf{p})-R(\boldsymbol{\nu})
$$

Proposition 6.1: (Optimal price per honored demand) If $F$ is given by (3) then under Assumption 3 an inner solution $p_{i}^{*}$ of the licensee's problem (4) satisfies for all $i \in L$

$$
p_{i}^{*}=\left(1+\varepsilon_{i}^{-1}\left(p_{i}^{*}\right)\right)^{-1} \sum_{j \in N} w_{i j} \frac{d}{d \kappa_{j}} U\left(\boldsymbol{\lambda}\left(\mathbf{p}^{*}\right)\right) .
$$

Note that here the form of the optimal price does not include a discount at the acceptance probability as the price per demand case since the price is already applied to accepted calls.

\section{Computation of Optimal PRices}

The sensitivities $\frac{d}{d \kappa_{j}} Q(\boldsymbol{\lambda}(\mathbf{p}))$ in (10) and $\frac{d}{d \kappa_{j}} U(\boldsymbol{\lambda}(\mathbf{p}))$ in (11) possess certain properties that can be useful in computing optimal price $\mathbf{p}^{*}$. Consider first the latter quantity $\frac{d}{d \kappa_{j}} U(\boldsymbol{\lambda}(\mathbf{p}))$ where for notational convenience we denote it by $c_{j}(\mathbf{p})$. Notice that $c_{j}(\mathbf{p})$ is the reduction in the overall revenue of the licensee due to a unit reduction in the interference threshold of cell $j$. To obtain a more explicit characterization of this quantity, let $\eta_{j}(\mathbf{p})$ denote the unit increase in the unit blocking probability $b_{j}(\boldsymbol{\lambda}(\mathbf{p}))$ at cell $j$ per unit decrease in the interference threshold of the cell. Therefore the intensity of calls at cell $i$ after being thinned due to blocking at cells other than $j$ can be written as

$$
\rho_{i j}(\mathbf{p})=\lambda_{i}\left(p_{i}\right)\left(1-b_{j}(\boldsymbol{\lambda}(\mathbf{p}))\right)^{-1} \prod_{k}\left(1-b_{k}(\boldsymbol{\lambda}(\mathbf{p}))\right)^{w_{i k}} .
$$

In fact, in the above expression the call is not considered thinned at cell $j$ because all the call units are thinned at that cell except one. Such a call is accepted on cell $i$ with probability $1-b_{j}(\boldsymbol{\lambda}(\mathbf{p}))$ in which case it returns revenue $r_{i}\left(p_{i}\right)$. Note that a unit decrease in $\kappa_{j}$ has the effect of increasing the unit blocking probability $b_{j}(\boldsymbol{\lambda}(\mathbf{p}))$ by $w_{i j} \eta_{j}(\mathbf{p})$ units. The reduction in revenue due to such calls is then given by

$$
\left(1-b_{j}(\boldsymbol{\lambda}(\mathbf{p}))\right)^{-1} \sum_{i \in N} \rho_{i}(\mathbf{p}) w_{i j} \eta_{j}(\mathbf{p}) r_{i}\left(p_{i}\right),
$$

where

$$
\rho_{i}(\mathbf{p})=\left(1-b_{j}(\boldsymbol{\lambda}(\mathbf{p}))\right) \rho_{i j}(\mathbf{p})
$$

is the rate of accepted calls at cell $i$. By way of blocking in its neighborhood, a call has further consequences in operation of other cells. Since each blocked call in cell $i$ can be associated with increasing the threshold of each other cell $l$ by $w_{i l}$ units and cell $j$ itself by $w_{i j}-1$ units, a unit decrease in $\kappa_{j}$ leads to an increase of

$$
\begin{array}{r}
\left(1-b_{j}(\boldsymbol{\lambda}(\mathbf{p}))\right)^{-1} \sum_{i \in N} \rho_{i}(\mathbf{p}) w_{i j} \eta_{j}(\mathbf{p}) \times \\
\left(\left(w_{i j}-1\right) c_{j}(\mathbf{p})+\sum_{l \in N-j} w_{i l} c_{l}(\mathbf{p})\right)
\end{array}
$$

in the revenue obtained from all the cells in the network. Therefore $c_{j}(\mathbf{p})$ can be written in the form

$$
\begin{aligned}
c_{j}(\mathbf{p})= & \varphi_{j}(\mathbf{p}) \sum_{i \in N} w_{i j} \rho_{i}(\mathbf{p}) \times \\
& \left(r_{i}\left(p_{i}\right)-\left(w_{i j}-1\right) c_{j}(\mathbf{p})-\sum_{l \in N-j} w_{i l} c_{l}(\mathbf{p})\right),
\end{aligned}
$$

where $\varphi_{j}(\mathbf{p})=\eta_{j}(\mathbf{p})\left(1-b_{j}(\boldsymbol{\lambda}(\mathbf{p}))\right)^{-1}$.

A similar relation can be written for the derivatives $\frac{d}{d \kappa_{j}} Q(\boldsymbol{\lambda}(\mathbf{p}))$ as well. In fact the only distinction is to assume that $Q(\boldsymbol{\lambda}(\mathbf{p}))$ is the revenue from the whole network when $r_{i}\left(p_{i}\right)$ for all $i \in L$ are taken to be 0 .

Moreover, it can be shown that for a fixed value of $\mathbf{p}$ the above relations identify the values $c_{j}(\mathbf{p})$ uniquely [14]. Also in cases when an inner solution $\mathbf{p}^{*}$ exists, properly damped versions of the recursion

$$
\begin{aligned}
p_{i}^{k+1}= & \left(1+\varepsilon_{i}^{-1}\left(p_{i}^{k}\right)\right)^{-1} \sum_{j \in N} w_{i j} c_{j}^{k} \\
c_{j}^{k+1}= & \varphi_{j}\left(\mathbf{p}^{k}\right) \sum_{i \in N} w_{i j} \rho_{i}\left(\mathbf{p}^{k}\right) \times \\
& \left(r_{i}\left(p_{i}^{k}\right)-\left(w_{i j}-1\right) c_{j}^{k}-\sum_{l \in N-j} w_{i l} c_{l}^{k}\right)
\end{aligned}
$$

may converge, thereby yielding $\mathbf{p}^{*}$. In fact, while we don't provide a convergence argument for this recursion, in Section VIII we give a numerical example where the recursion indeed converges.

\section{NUMERICAL STUDY}

\section{A. Computation of Optimal Prices Using Recursion}

We start our numerical study by showing an example where recursion (12), (13) introduced in section VII proves useful in computing optimal prices. We do first an exhaustive search 


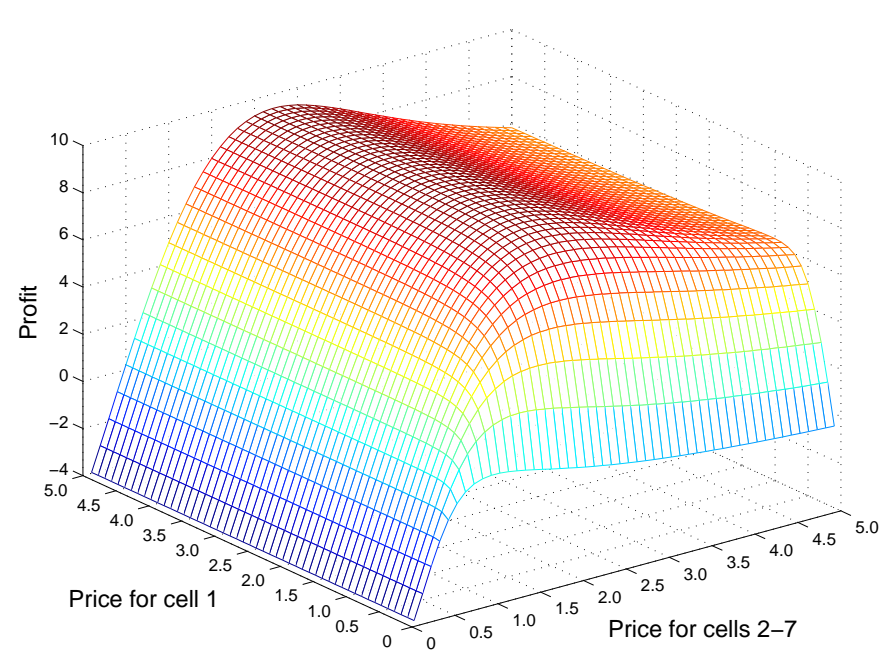

Fig. 2. Profit of the licensee from leasing cells $1-7$ in the network shown in Figure 1 using per honored demand pricing. Call demand on the leased cells is taken to be as in (15). $\kappa_{i}=5.0, w_{i j}=0.5, w_{i i}=1.0$, and $\nu_{i}$ as given in (14) for all $i, j$.

for prices that maximize the profit of the licensee from the network. Then we use the recursion to compute prices and compare the results.

Namely, consider a 19-cell hexagonal lattice topology with the corresponding network graph shown in Figure 1. The cells are assumed to have equal interference thresholds, that is, $\kappa_{i}=$ 5.0 for $i \in N$. We shall assume that a call generates half of the interference in neighboring cells relative to its own cell. More specifically, $w_{i j}=0.5$ for each edge such that $i \neq j$ and $w_{i i}=1.0$ for all nodes $i$. We are interested in computing price per honored demand for leasing cells $1-7$. The traffic demand of the licensee prior to the transaction is taken in call per unit time as

$$
\nu_{i}= \begin{cases}0 & \text { if } i=1, \ldots 7 \\ 1 & \text { if } i=8, \ldots 19\end{cases}
$$

On the other hand, call demand by the lessee is assumed to be following the pattern

$$
\alpha_{i}\left(p_{i}\right)=\beta_{i} p_{i}^{-2} \quad i=1, \ldots 7 .
$$

where

$$
\beta_{i}= \begin{cases}1 & \text { if } i=1 \\ 5 & \text { if } i=2, \ldots 7 .\end{cases}
$$

In other words cells $2-7$ experience the same demand which is different from the demand on cell 1 . Therefore by spatial and parameter symmetry of the problem, cells $2-7$ should have the same price, and so there are at most two distinct cell price values. We show the profit of the licensee in Figure 2 for different price values for cells 1 and cells $2-7$. Profit values represented in the figure are computed for price step of 0.1 on each axis. The highest profit is 9.42 achieved at $p_{1}^{*}=2.9$ and $p_{i}^{*}=2.2$ for $i=2, \ldots 7$.

Next we compute prices for the same setup using a damped version of recursions (12), (13) and show the convergence

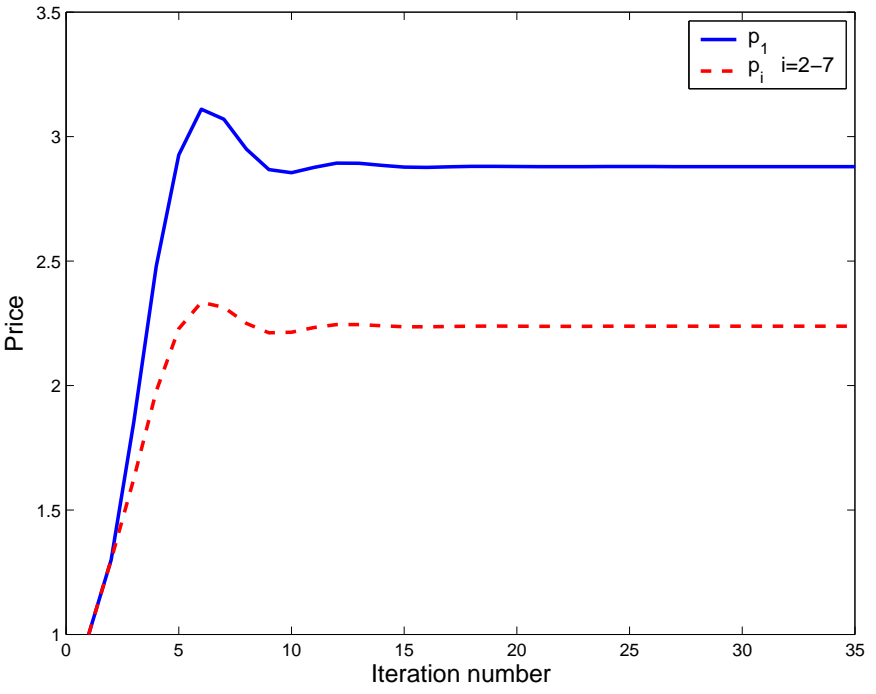

Fig. 3. Convergence paths for prices of cells $1-7$ when applying per honored demand pricing in the network in Figure 1. Call demand on the leased cells is taken to be as in (15). $\kappa_{i}=5, w_{i j}=0.5, w_{i i}=1.0$, and $\nu_{i}$ as given in (14) for all $i, j$. The limits agrees with the optimal price observed from Figure (2).

paths of the prices in Figure 3. The resulting price values are $p_{1}^{*}=2.88$ and $p_{i}^{*}=2.24$ for $i=2, \ldots 7$.

As can be seen the recursively computed limits agree with the results from the exhaustive search. In fact prices converge in a relatively short period in less than 20 iterations.

\section{B. Cell vs. Region Pricing}

The network effect of interference due to a call admitted in a certain cell depends in fact on the location of that cell. For example consider the network represented by the graph in Figure 1. The interference from a call admitted in cell 1 affects the call occupancy of neighboring cells $2-7$, while a call admitted in cell 8 affects only cells $2,9,19$. Therefore efficient pricing should take into consideration geometric location of the cells and their proximity to the other cells in the network.

To support this argument we consider the same lattice model represented in Figure 1. The capacities of the cells are taken to be equal with value $\kappa_{i}=5.0$ and all non-zero edge weights are also equal with value $w_{i j}=0.5$ and $w_{i i}=1.0$ for all $i, j$. We assume pricing per honored demand for leasing cells $1-7$. The demand is as given in (15) but $\beta_{i}=1$ for all $i$. First we solve numerically for the profit maximization problem (4) that seeks optimal price for each cell $1-7$. Namely we employ recursion (12), (13) to compute optimal prices. We show the corresponding values of the profit from the lease in Figure 4 for different traffic loads on the retained region; i.e. cells $8-19$. On the other hand we consider the approach in [11] (Proposition 6.1) where the whole subregion, i.e., cells $1-7$, is priced by a scalar quantity. We employ the same recursion and show the profit values in Figure 4. As can be noticed from the figure more profit is obtained when the pricing problem is further broken to the cell level. In other words, exploiting the spatial effect of interference due 


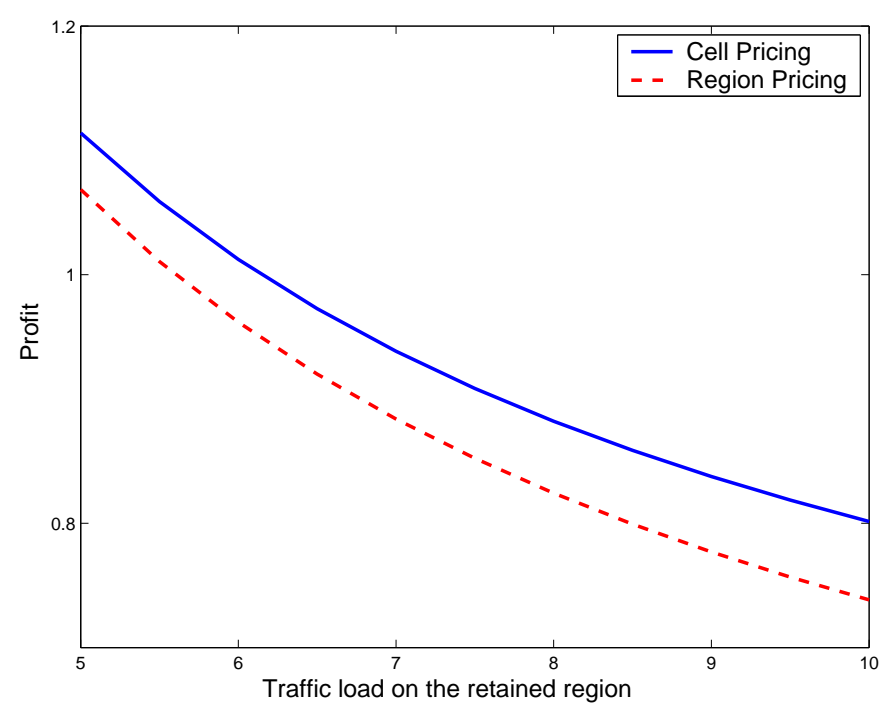

Fig. 4. Profit gained from leasing cells $1-7$ in the network shown in Figure 1 for different traffic loads on the retained region and when implementing per honored demand pricing. Call demand is given in (15) with $\beta_{i}=1$ for $i=1, \ldots, 7 . \kappa_{i}=5.0, w_{i j}=0.5, w_{i i}=1.0$ for all $i, j$. Profit when pricing per cell is shown to be better than the case when the whole region is priced by a scalar quantity.

to geographical locations of the cells helps gain more profit.

\section{CONCLUSION}

We considered the problem of optimal pricing for secondary access of spectrum in CDMA-based cellular wireless networks. We presented a network operational model and formulated the profit maximization problem of the primary license holder. We took into account the network-wide effect of interference and provided a form of optimal prices based on first order optimality analysis. Optimal prices are shown to depend on the extent of the generated interference of accepted calls. The from of the prices suggests that the amount charged per admitted call should balance the corresponding loss of revenue incurred by the licensee due to the influence of the call. We also presented a recursive technique for computing optimal prices and numerically solved example problems.

While the technical focus of this paper is on networks that employ CDMA as spectrum access mechanism, narrowband networks, in which a channel cannot be utilized simultaneously in neighboring cells, generally appear harder to analyze due to combinatorial consequences of interference. In fact the techniques presented in this paper apply to certain narrowband topologies and channel assignment policies. However a general treatment of such networks can be a future direction for this work.

\section{ACKNOWLEDGMENT}

This work was funded in part by NSF through grants ANI0238397, CCF-0430983, CNS-0435353, ANI-0132802, ANI0240333 , CNS-0435312.

\section{REFERENCES}

[1] The Defense Advanced Research Projects Agency (DARPA), The Next Generation Program. http://www.darpa.mil/sto/smallunitops/xg.html.

[2] The Federal Communications Commission, Secondary Markets Initiative. http://wireless.fcc.gov/licensing/secondarymarkets/.

[3] H. R. Varian, Microeconomic Analysis, Norton, New York, 1984.

[4] A. Tonmukayakul and M. Weiss, "Secondary use of radio spectrum: a feasibility analysis," Telecommunications Policy Research Conference, 2004.

[5] C. Courcoubetis and R. Weber, Pricing Communication Networks: Economics, Technology and Modelling, Wiley, New York, 2003.

[6] S. L. Hew and L. B. White, "Interference-based dynamic pricing and radio resource management for WCDMA networks," Vehicular Technology Conference, 2005.

[7] J. M. Peha and S. Panichpapiboon, "Real-time secondary markets for spectrum," Telecommunications Policy Journal, August 2004.

[8] J. Ni and S. Tatikonda, "Revenue optimization via call admission control and pricing for mobile cellular systems," IEEE International Conference on Communications (ICC), 2005.

[9] C. U. Saraydar, N. B. Mandayam, and D. J. Goodman, "Pricing and Power Control in a Multicell Wireless Data Networks," IEEE Journal on Selected Areas in Communications, vol. 19, No. 10, pp. 1883-1892, 2001.

[10] J. Huang, R. A. Berry, and M. L. Honig, "Auction-based spectrum sharing," Mobile Networks and Applications, vol. 11, pp. 405-418, 2006.

[11] A. Al Daoud, M. Alanyali, and D. Satrobinski "Secondary Pricing of Spectrum in Cellular CDMA Networks," IEEE Symposium on New Frontiers in Dynamic Spectrum Access Networks (DySPAN), April 2007.

[12] D. Everitt, "Analytic traffic models of CDMA cellular networks," International Teletraffic Conference ITC-14, 1994.

[13] F. P. Kelly, "Loss networks," Annals of Applied Probability, vol. 1, pp. 319-378, 1991.

[14] F. P. Kelly, "Routing in circuit-switched networks: Optimization, shadow prices and decentralization," Advances in Applied Probability, vol. 20, pp. 112-144, 1988. 